\title{
Sprachen-Kontakt und regionale Schriftsprachlichkeit Bohemismen im Prager Standarddeutsch um 1920
}

\section{Boris Blahak}

In the early $20^{\text {th }}$ century the term 'Prague German' was used to classify the German sociolects which were spoken by the underclasses of Prague containing numerous Czech interferences. Looking for (social) delimitation the German-speaking bourgeoisie of Prague distinguished themselves from these idioms and stressed (in an exaggerating way) the special 'purity' of their own group language German. However, when looking into Franz Kafka's writings one comes across numerous phenomena obviously following Czech patterns. The study tries to clarify whether these phenomena reveal an influence of the Prague sociolects on the (allegedly isolated) language of the Prague German educated classes. For this reason, the German of writers coming from Kafka's environment is examined for similarities. At the same time shifting focus on other contemporary print media of Prague (newspapers, dictionaries) the question will be answered whether such phenomena diachronically prove to be even part of a regional standard language.

Franz Kafka - language attitudes - Prague standard variety of German - fiction - daily press dictionaries - Bohemisms - language contact - regional written language

Mit dem Terminus ,Prager Deutsch' bezeichnete man im frühen 20. Jh. die deutschen Soziolekte der Prager Unterschichten, die zahlreiche tschechische Interferenzen aufwiesen. Bemüht um (soziale) Abgrenzung distanzierte sich das deutschsprachige Prager Bürgertum von diesen Idiomen und wies hierzu (überspitzt) auf die besondere ,Reinheit' der eigenen Gruppensprache Deutsch hin. Beim Blick in Franz Kafkas Schriften stößt man jedoch auf zahlreiche Erscheinungen, die scheinbar tschechischem Muster folgen. Die Untersuchung versucht zu klären, ob diese Phänomene einen Einfluss der Prager Soziolekte auch auf die (angeblich abgezirkelte) Sprache der Prager deutschen Bildungsschicht dokumentieren. $\mathrm{Zu}$ diesem Zweck wird auch das Deutsch von Schriftstellern aus Kafkas Umfeld auf Übereinstimmungen untersucht. Zugleich soll ein Blick auf andere zeitgenössische Prager Printmedien (Tageszeitungen, Wörterbücher) die Frage beantworten, ob sich solche Phänomene diachron sogar als regional standardsprachlich erweisen.

Franz Kafka - Sprach-Attitüden - Prager Standardvarietät des Deutschen - Belletristik Tagespresse - Wörterbücher - Bohemismen - Sprachen-Kontakt - regionale Schriftsprachlichkeit

\section{1. ,Prager Deutsch‘ und ,horror bohemismorum': sozial geprägte Sprach- Attitüden in Prager deutschsprachigen Schriftstellerkreisen um 1920}

In seiner Historischen Lautgeographie des gesamtbairischen Dialektraumes machte Eberhard Kranzmayer 1956 folgende Feststellung über das im 19. Jh. in Prag gesprochene Deutsch: 
Das Pragerdeutsch galt bis gegen Ende des vorigen Jahrhunderts für die Nieder- und Oberösterreicher und für die Wiener als das schönste Deutsch auf dem Boden der alten österr.-ungar. Monarchie; [...] mit Recht. Es hat sich [...] die Sprechweise unter der ständigen Einwirkung der vielen Zuwanderer aus den verschiedensten deutschen Sprachlandschaften möglichst aller lokalen Dialektschattierungen entäußert (Kranzmayer 1956: 80).

Kranzmayer kolportierte hier ein Autostereotyp, das sich unter Angehörigen des Prager deutschsprachigen Bürgertums in der Diaspora bis weit in die zweite Hälfte des 20 Jh. hielt, als die multilinguale Prager Sprachlandschaft der k. u. k. Zeit längst nicht mehr existierte. Es besagte, in Prag seien gesprochene und geschriebene Sprache deckungsgleich gewesen, nämlich ein Standarddeutsch bar jeder regionalen Tönung. Johannes Urzidil (1972: 209) brachte dieses Bild auf den Punkt, als er für die Schriftsteller seines sozialen Umfeldes kollektiv in Anspruch nahm, sie schrieben ihre Prosa in derselben Sprache, die sie tagsüber sprächen, und diese Sprache sei „das reine und unversetzte Hochdeutsch“, gleichsam „die deutsche Sprache.“ „Wenn wir keine deutschen Autoren mehr sind“, so Urzidil, „dann ist es niemand.“

Kranzmayer übernahm diese Darstellung zwar; allerdings verwendete er für die Sprache der Prager Oberschicht einen Terminus, von dem sich gerade jene Autoren, die Urzidil angesprochen hatte, betont distanzierten. Unter ,Prager Deutsch' verstanden diese nämlich die Soziolekte der städtischen Unterschichten, die von tschechischen Sprachmustern überlagert gewesen seien, gesprochen von unvollkommen bilingualen Tschechen oder von Deutschen, „die von kindesbeinen an neben der čechischen sprache nur ein vom čechischen lautlich beeinflußtes deutsch sprechen hörten“" (Ritschel 1893: 129). In höchster Intensität sei dieses Idiom auf der Prager Kleinseite (Malá strana) verbreitet gewesen, weshalb man synonym auch vom „Kleinseitner Deutsch“ (Kisch 1992: 248) sprach.

Von diesen defizitären „Stigma-Soziolekten“ (Steinig 1980: 107) glaubte sich das (jüdisch-)deutsche Prager Bürgertum vehement abgrenzen zu müssen und wies hierzu immer wieder überspitzt auf die besondere ,Reinheit' der eigenen Gruppensprache Deutsch hin. Für Pavel Eisner (1946: 490) etwa war das, Prager Deutsch“ nichts anderes als ein "místní jazykový zmetek, zrozený ze špatného vzdělání a mizernějšího smýšlení. “1 Egon Erwin Kisch (1917: 4) polemisierte gegen „jenes deutsch-tschechische Kauderwelsch [...], das keine Sprache, sondern eben Prager Deutsch ist“; und auch Max Brod (1960: 219) hob ostentativ hervor, ,Prager Deutsch ' werde „von Tschechen, die sich deutsch ausdrücken wollten, gesprochen [...], kaum aber von Deutschen."

1 Dt. „örtlicher sprachlicher Bastard, hervorgegangen aus schlechter Bildung und noch erbärmlicherer Gesinnung" [Übers. d. Verf.]. 
Solche Aussagen scheinen durch das Bewusstsein geprägt, sich als Minorität in einer sprachsoziologischen Sondersituation ${ }^{1}$ positionieren zu müssen, in der Sprache und soziale Schicht entscheidende Parameter der Gruppenidentität und -exklusivität darstellten: Denn durch einen überwiegend tschechischen Zuzug während der Industrialisierung, durch sich häufende Loyalitätswechsel von der deutschen zur tschechischen Sprachgemeinschaft und durch eine höhere natürliche Wachstumsrate der tschechischen Bevölkerung war der Anteil der deutsch deklarierten Bewohner Prags an der Stadtbevölkerung bis 1900 auf 7,7 \% gesunken. Die Abgrenzung von der tschechischen Mehrheit wurde dabei maßgeblich durch soziale und wirtschaftliche Faktoren bedingt: denn etwa $70 \%$ der deutschsprachigen Prager gehörten dem Besitz- und Bildungsbürgertum an; demgegenüber stellten fast $50 \%$ der tschechischsprachigen Stadtbewohner Arbeiter und Tagelöhner (vgl. Cohen 1981: 92-93, 100-107).

Angesichts dieser Verhältnisse wurde in derselben Schicht, konträr zur Abqualifizierung des ,Prager Deutsch', das Autostereotyp der Mustergültigkeit des muttersprachlichen Deutsch in Prag beschworen (vgl. z. B. Stein 1976: 15). Egon Erwin Kisch (1917: 4) etwa hob die ,Reinheit' der Muttersprache nahezu ins Sakrale, als er dafür plädierte, es müsse ,zuhause von Kindheit an die Heiligkeit der Sprache hochgehalten" werden; und Max Brod sah in der Übernahme tschechischer Sprachmuster gar einen ,Sündenfall' gegenüber der eigenen Sprachgemeinschaft:

In den deutschsprechenden Schichten Prags, die deutsche Schulen besucht hatten, wurden jene Anklänge an das Tschechische [...] immer strengstens als grobe Fehler, als „Pragismen“ oder „Tschechismen“ verfolgt und ausgemerzt, ja sogar noch besonders empfindlich verlacht. [S]o muss man wissen, daß es nie einem gebildeten Deutsch-Prager eingefallen wäre, sich eines solchen „Angleichens an die Sprache der Nachbarn“ schuldig zu machen (Brod 1960: 220).

Solche überzogenen Aussagen lassen ihre Verfasser allerdings geradezu als Gewährsleute für das kollektive Wissen der (jüdisch-)deutschen Prager Bildungsschicht von den Auswirkungen des deutsch-tschechischen SprachenKontakts auf die eigene Gruppensprache erscheinen. Denn offenkundig liegt ihnen der latente eigene Verdacht zugrunde, sich in Prag im Laufe der muttersprachlichen Sozialisierung womöglich tatsächlich ungesteuert ein Idiom angeeignet zu haben, das aus Sicht der schulisch propagierten Klassiker-Sprache ${ }^{2}$ Mängel aufwies: durch

$1 \mathrm{Zu}$ den in tschechischen Kreisen verbreiteten negativen Attitüden gegenüber den deutschsprachigen Bewohnern Prags, namentlich den Juden unter ihnen, als Teil einer konstanten sozialen und psychologischen Situation zu Kafkas Lebzeiten s. u. a. Braese (2010), Kučera (2013; 2014) und Blahak (2014a).

2 Um das Deutsche „als eine zur Klassizität ausgebildete Sprache und somit prestigebesetzte Ersatzvarietät humanistisch zu konnotieren“" (Ziegler 1999: 93), wurde die anvisierte Schriftsprache im 19. Jh. im deutschen Sprachraum schulisch anhand des Sprach- und Stilideals der Klassiker (Goethe, Schiller) vermittelt. Dass dies auch für die deutschsprachigen Kreise in Prag galt, lässt sich anhand der Schulgrammatik (Kummer ${ }^{3}$ 1892) nachvollziehen, auf dessen Grundlage Kafkas Deutschunterricht am Staatsgymnasium mit deutscher Unterrichtssprache in Prag-Altstadt erfolgt war. 
den Kontakt mit der Mehrheitssprache Tschechisch und dem ,fehlerhaften' Deutsch, das man v. a. auf der Kleinseite hörte und dessen Verwendung man daher umso vehementer bestritt. Das Autostereotyp der sprachlichen Makellosigkeit scheint mithin in ursächlicher Beziehung zu den Sprachen-Kontakt-Phänomenen im ,Prager Deutsch`zu stehen, indem es das Bewusstsein ihrer Alltagspräsenz dokumentiert. Aus literatur- und sprachsoziologischer Sicht hat man es somit mit einer kollektiven Wunsch-Projektion zu tun (vgl. Binder 1996: 208-209).

Um dieses Bild zu untermauern, verwiesen Brod (1969: 149-150) und Urzidil (1972: 208) im Rückblick immer wieder auf die Literatursprache Franz Kafkas, die ja bereits von bekannten Zeitgenossen als „vorbildliche[s]“ (Tucholsky 1962: 473) und „klassische[s] Deutsch“ (Hesse 1987: 487) beurteilt worden war. Die Rezeptionsgrundlage solcher Aussagen stellte allerdings die zunächst einzige verfügbare, durch Max Brod unkritisch eingerichtete Werkausgabe dar. Brod war bei seiner Textedition nicht als Philologe vorgegangen: Da in seinen Augen die Erfolgsaussichten von Kafkas Prosa entscheidend waren, war er bestrebt, regional markierte sprachliche Formen zu entfernen, welche die Lesbarkeit außerhalb Prags hätten erschweren können. Seine Vorgehensweise rechtfertigte er mit dem Entwurfscharakter der Handschriften. So seien „Flüchtigkeitsfehler stehen geblieben, die er [Kafka], wenn er das Buch selber ediert hätte, bei seiner großen Gewissenhaftigkeit gewiß verbessert hätte“ (Brod 1974: 352).

Erst angesichts der seit den 1950er Jahren zunehmenden Kritik an seiner Editionspraxis $^{1}$ räumte Brod schließlich notgedrungen, wenngleich unter dem Anschein der Beiläufigkeit, ein, er habe in Kafkas postumen Schriften durchaus „Pragismen', die eine Beeinflussung des deutschen Stils durch die tschechische Syntax darstellen“ (Brod ${ }^{3}$ 1954: 300), vorgefunden. Zugleich ergänzte er jedoch beschönigend, solche Anomalien seien als

[v] ereinzelte Fehler aus Unachtsamkeit [...] unter dem Einfluß der tschechischen Umwelt in der deutschen Umgangssprache und im deutschen Schrifttum Prags in ganz seltenen Ausnahmefällen [...] vorgekommen[,] beispielsweise jene wenigen Sprachunrichtigkeiten in den Manuskripten Kafkas, die ich bei der Drucklegung richtiggestellt habe, weil ich weiß, daß auch mein Freund, hätte er je an eine Drucklegung gedacht, diese Korrekturen unbedingt vorgenommen hätte (Brod 1969: 150) [Herv. d. Verf.].

\section{Das ,Deutsch der Prager Deutschen` auf dem Prüfstand: Untersuchungsziele, -korpus und -methode}

Bemerkenswerterweise haben sich auch Vertreter der germanistischen und slavistischen Sprachwissenschaft, etwa Skála (1977: 274), Thieberger (1979: 178) und Nekula (2004: 96), bis heute skeptisch gegenüber der Vorstellung geäußert, der

1 Zu dieser s. bes. Beißner (1952), Uyttersprot (1957), Martini (1958) und Dietz (1963). 
deutsch-tschechische Sprachen-Kontakt könnte sich in Prag auch auf das Deutsch der Prager Bildungsschicht in wahrnehmbarer Weise ausgewirkt haben. Grundlegende Untersuchungen hierzu wurden allerdings bisher nicht vorgelegt. Will man sich selbst ein Bild verschaffen, so scheint ein Ansatz beim Prosa-Deutsch Franz Kafkas tatsächlich nahe liegend: Mit deutscher Muttersprache und strenger deutscher Schulbildung entstammte er dem gleichen sprachlichen Sozialisationsmilieu wie etwa Brod, der das Deutsch seines Freundes einerseits normalisierend überarbeitet hatte und es andererseits dann als exemplarisch für die gemeinsame, angeblich ,makellose‘ Gruppensprache deklarierte.

Verfolgt man das Ziel, Kafkas Deutsch auf tschechische Sprachstrukturen hin zu überprüfen, so erhält man am ehesten durch die im S. Fischer-Verlag (Frankfurt a. M.) seit 1982 erscheinende Kritische Kafka-Ausgabe (KKA) ein authentisches, transparentes Bild von Kafkas Autograph: In ihr wird jedem Textband, der Kafkas Prosa in lesbarer Form wiedergibt, ein Apparatband zugeordnet, der neben sämtlichen Textvarianten Kafkas auch die editorischen Eingriffe seiner Herausgeber verzeichnet. Da Kafkas Varianten meist Sofortkorrekturen darstellen, bilden sie den Text sozusagen ,in statu nascendi' ab (vgl. Pasley ${ }^{2} 1983: 73$, 76-77; Schillemeit 2002: 83-84) und erlauben häufig Rückschlüsse darauf, wie Wort- oder Satzbestandteile ursprünglich gedacht und unwillkürlich (z. T. substandardlich) verschriftlicht wurden, bevor der Autor sich selbst korrigierte. ${ }^{1}$

In der Frage der Gruppenspezifik tschechischer Interferenzen wird im Weiteren das Prosa-Deutsch von Schriftstellern aus Kafkas engerem Umfeld, deren (Sprach-) Biographien Parallelen zu seiner eigenen aufweisen, auf Übereinstimmungen untersucht. Ein gruppenspezifisches Auftreten kann zugleich die bei literarischer Sprachverwendung immer gegebene Möglichkeit relativieren, tschechische Sprachmuster könnten allein dem kreativen Umgang eines Dichters mit der Sprache entspringen, und sicher stellen, dass es sich um ,echte Bohemismen' handelt, die Teil des (auch alltäglichen) Prager Sprachgebrauchs waren. Als erstes Kontrollkorpus dienen im Rahmen des folgenden Vergleichs Max Brods Reisetagebücher (Brod/Kafka 1987), seine Romane Jüdinnen (1911) und Arnold Beer (1912), seine Novelle Die erste Stunde nach dem Tode (1916) sowie sein Essayband Über die Schönheit häßlicher Bilder (1913), ferner Egon Erwin Kischs Roman Der Mädchenhirt (51922) und sein Kriegstagebuch Soldat im Prager Korps (1922) sowie Otto Picks Novellensammlung

1 Das Untersuchungskorpus bilden im Folgenden Kafkas literarische Schriften und Tagebücher, deren Edition bereits abgeschlossen ist. Seiten-/Zeilen-Angaben aus der KKA erfolgen im Weiteren nach Kafka (21983), abgek. als S, Kafka (1990a), abgek. als T, Kafka (1990b), abgek. als P, Kafka (1992), abgek. als N2, Kafka (1993), abgek. als N1, Kafka (1994/1996), abgek. als D, und Kafka (2002), abgek. als V. Ein ,e' vor der Angabe verweist auf editorische Eingriffe, ein ,v' bezeichnet Varianten Kafkas, wiedergegeben durch die Zeichen $[a]$ : Streichung von $a$; $\{b\}$ : Einfügung von $b$; $(e>i)$ : Überschreibung von $e$ zu $i ; c^{A}$ : Ansatz zum Buchstaben $c$. Fehlende', durch Editoren ergänzte Textbestandteile werden an entsprechender Stelle recte in nach außen gekehrten Winkelklammern ( zur) gesetzt. Textzitate erfolgen kursiv. 
Die Probe (1913). Zugleich soll ein Blick auf andere zeitgenössische Prager Druckerzeugnisse, die zu den örtlichen normsetzenden Instanzen gehören, die Frage klären, ob sich solche Phänomene diachron $u$. U. regional sogar als standardsprachlich erweisen. Hierzu werden als weitere Kontrollkorpora ausgewählte Ausgaben der Prager Presse (PP 1921a-c) und des Prager Tagblatts (PT 1921) aus dem Jahre 1921 herangezogen sowie fünf einschlägige in Böhmen erschienene Wörterbücher (Kott 1878-1893; Herzer und Prach 1909-1920; Sterzinger 1916-1935; Siebenschein 1936-1948; Kumprecht ${ }^{3} 1940$ ), welche die Zeitspanne von Kafkas Geburt bis zum Ende der deutschsprachigen Gemeinde Prags abdecken ${ }^{1}$

\section{3. ,Kleinseitner' Phrasenstrukturen in Franz Kafkas Deutsch und ihre Analogien in Prager Referenzquellen}

\subsection{Elliptische syntaktische Strukturen mit Parallelen zum Tschechischen}

Überprüft man Kafkas Varianten nun im Hinblick auf tschechische Sprachstrukturen, so lassen sich diese im Bereich der Syntax etwa im häufigen Ausfall zweier Wortarten ausmachen, die das Tschechische nicht kennt. Von Ellipse betroffen ist zunächst die Infinitivkonjunktion $z u$ :

Grundregel [...], sich niemals überraschen $\mathrm{zu}$ < lassen (Pe.222/13-15), ob es (s>r)ichtig war, so $\{z u\}$ handeln (Pv.225/4-5), [einer] [\{sie\}] suchte [den] \{ein\}ander $[n]$ zurückrzuddrängen (Sv.125/27-126/1), was sollte uns zwingen Sie auf( $\left.n^{4}>z\right)$ unehmen (Sv.153/7-8), um Dein Mitleid hervor(lo>zu)locken (Sv.250/16-17), da ich so da(rum>nach) verlangte, zurückrzu'kehren (Vv.126/19-20), solche Maßnahmen gegen zwei kleine Bälle $\{z u\}$ ergreifen (N1v.234/14-15), um [...] si(e>ch) für die Dämpfung durch den Teppich $\{z u\}$ entschädigen (N1v.238/5-7), Es ist mein Recht, solche Formalitäten $\{z u\}$ erfinden (N1v.270/3-6(1)19*-20*). ${ }^{2}$

Dabei zeichnet sich durch folgende Indizien eine gewisse Gruppenspezifik und gemäßigte Standardnähe für Prag ab: Ähnliche $z u$-Ellipsen finden sich durchaus verstreut im Prager Tagblatt wie in den Schriften Max Brods, Egon Erwin Kischs und Otto Picks:

1 Die Festlegung der genannten Kontrollkorpora orientiert sich an dem von Ulrich Ammon (2005) entworfenen Konzept von Standardvarietät, das von einem dynamischen Diskurs ausgeht, in dem sprachliche Normen in einem sozialen Kräftefeld interaktiv verhandelt werden. Wörterbücher gehören dabei der Gruppe der Sprachkodizeslautoritativen Nachschlagewerke an, Erzeugnisse der Tagespresse und Texte von Autoren konzeptioneller Schriftlichkeit jener der Modellsprecher/-schreiber, die Ammon (neben Sprachexperten und Normautoritäten) zu den vier einflussreichen Gruppen im sozialen Kräftefeld der Standardvarietät(en) einer Sprache zählt.

2 Vergleichbare $z u$-Ellipse sind in Fällen von Kontrastnivellierung in Texten tschechischer Deutschsprecher zu erwarten (vgl. Rinas 2003: 162-163; Štícha 2003: 395-396, 673-674). Zur Diskussion des Phänomens bei Kafka s. Blahak (2015: 516-521). 
nichts weiteres hinzurzu fügen habe (PT 1921: 2), Er half ihr schnell, die Fäden loszu $b i n d e n$ (Brod 1911: 173), ob sie Tee oder Kaffee szu trinken wünschen (Kisch 1922: 212), Ich komme mich von dir rzu< verabschieden (Pick 1911: 72).

Darüber hinaus entfernte die Deutsche Montags-Zeitung (Prag) bei der Veröffentlichung von Kafkas Erzählung Zum Nachdenken für Herrenreiter ein an sich normkonformes $z u$, obwohl dieses in der Leipziger Ausgabe der Betrachtung (Kurt Wolff) bereits in gedruckter Form vorlag: und doch nicht weiß, was anzufangen (Dv.31/17).

Die zweite vom Ausfall betroffene Wortart sind obligatorische Artikel. In Kafkas Prosa-Handschriften finden sich hierzu u. a. folgende Beispiele:

die er in der Hand hielt (Pe.13/25), \{in einen Winkel bei , der Tür\} (Pv.70/12), nur zu $\{m\}$ Fabrikanten (Pv.175/5-6), bis auf $\{$ den $\}$ Hinauswurf (Sv.118/20), durch \{die\} Art ihrer Antworten (Sv.183/18(1)28*-29*), Seine [Mü] (ku> ha) lbkugelige Mütze reichte bis zu \{den\} Brauen (N1v.15/24-25), waren sie oft bis zurr Tür entgegengelaufen (N1e.259/27-260/1), durch \{das\} Labyrint (N2v.622/1-2), es klang durchıs ganze Treppenhaus (Dv.139/26), auf\{s\} höchste beleidigt (Dv.178/12). ${ }^{1}$

Dass diese ,sparsame' Artikelverwendung, die schon August Schleicher (1851) in Böhmen aufgefallen war, allerdings ein (sogar standardnahes) gruppenspezifisches Phänomen der deutschsprachigen Prager war, zeigen vergleichbare Belegstellen in der Prager Tagespresse - und nicht zuletzt wiederum in der Prosa Brods, Kischs und Picks:

in der Steiermark (PT 1921: 15), gegen ein verbohrtes Schulsystem (PP 1921a: 6), was 'das`Zeug hält (Brod 1911: 107), um den stärkeren Buben in ,den Popo zu zwicken (Kisch 1922: 142), vonx ersten bis zum letzten Buchstaben (Pick 1913: 31).

Auch das indefinite Numerale paar, das von Kafka analog zum tschechischen Muster $\varnothing+p a ́ r+$ Substantiv (Gen. Pl.) meist mit Nullartikel ${ }^{2}-$ z. B. Fräulein Montag folgte ihm paar Schritte (P.323/24-25) - verwendet wurde, erweist sich nicht nur als gruppenspezifisch, sondern sogar als Teil des Prager Schriftdeutschen: Obwohl Brod (31954: 300) bemüht war, dieses aus seiner Sicht stigmatisierende ,Schibboleth' lückenlos aus Kafkas Schriften zu tilgen, verdeutlicht ein Blick in die Kontrollkorpora, dass nicht nur Otto Pick und Egon Erwin Kisch, sondern eben

1 Analoge Artikel-Ellipsen sind bei tschechischen Deutschsprechern in Fällen von Kontrastnivellierung zu erwarten (vgl. Skála 1991: 139). Zur Diskussion des Phänomens bei Kafka s. Blahak (2015: 354-358).

2 Die insgesamt 210 Belege von (ein) paar (,einige') in Kafkas Prosa und Tagebüchern lassen die deutliche Bevorzugung der Konstruktion ohne (160 Belege bzw. 76,2 \%) gegenüber derjenigen mit Artikel (50 Belege bzw. 23,8 \%) erkennen (vgl. Blahak 2014b: 45-46; 2015: 359-360). 
auch er selbst dieses paar literarisch zur Fingierung konzeptueller Mündlichkeit verwendet hatten:

erst paar Tage, paar Zeilen, paar Zeitschriften, paar Menschen (Pick 1913: 43-45, 73), für paar Minuten, Für lumpige paar Kronen (Kisch $\left.{ }^{5} 1922: 217,222\right)$, in so paar Seiten (Brod 1911: 265).

Unterstrichen wird die regionale Normkonformität der Konstruktion durch einen Eintrag bei Siebenschein (1944: 133): das bekommen Sie schon für p. Kronen. ${ }^{1}$

\subsection{Kollokationen/Präpositionalverwendungen nach tschechischem Muster}

Andere Bohemismen können im Bereich der Kollokation nachgewiesen werden; denn Kafkas Deutsch schwankt in identischen Konstruktionen wiederholt zwischen den Präpositionen an und auf - ein Verweis auf die Unterdifferenzierung des Tschechischen gegenüber dem Deutschen, das in den folgenden Textbelegen für beide Präpositionen nur ein Äquivalent besitzt, nämlich die Präposition $n a:^{2}$

$$
\begin{aligned}
& \text { an } \times \text { auf } \\
& \text { wenn man an die Türe schlug } \times \text { Warum } s\left(h^{A}>c\right) h l a g e n \text { Sie so verrückt auf } \\
& \text { (V.97/21). dieTür\{?\} (Vv.8/24-25). } \\
& \text { an der Wand das Bild } \times \text { Auf der Wand [...] ein kleines Bild } \\
& \text { (N2.76/7-8). (N1.312/10-11). } \\
& \text { Sterne am Himmel } \times \text { auf dem Himmel die Sterne } \\
& \text { (N1.191/ 17). (N1.142/23-24). } \\
& \text { am andern Ufer (N1.77/1). } \times \text { Auf beiden Ufern (N1.64/2-3). } \\
& \text { stützten si }(e>c) h[. . .] \text { an den Schreibtisch } \times \text { stützte sich [...] auf den Schreibtisch } \\
& \text { (D.165/11-12). (P.175/13-14). } \\
& \text { an einem Spritzenhebel gelehnt } \times \text { [auf seinen Besen ge(lehnt>stützt)] } \\
& \text { (S.299/11). (Sv.208/23). } \\
& \text { werden Sie in amtlicher Hinsicht } \times \text { indem sie Dich auf den } \\
& \text { ausschließlich an mich [...] verwiesen Gemeindevorsteher verwiesen } \\
& \text { (S.114/10-12). (S.363/2-3). }
\end{aligned}
$$

Beim Blick in Brods Kafka-Ausgabe fällt die geringe Zahl editorischer Eingriffe in diesem Bereich auf: Bei zwei Ausnahmen (Kafka 1953:165, 196) folgt sie anstandslos Kafkas Fassung letzter Hand. Dies spricht dafür, dass Brod diese

1 Zur varietätenlinguistischen Einordnung s. Blahak (2014b: 44-47; 2015: 358-362).

2 Bei der Übertragung von $n a$ als an oder auf führt diese Unterdifferenzierung bei tschechischen Deutschsprechern häufig zu analogen sprachkontrastiv bedingten Schwierigkeiten (vgl. Bednarský 2002; Štícha 2003: 574-575, 577-578, 581-582). Zur Diskussion des Phänomens bei Kafka s. Blahak (2015: 487-502). 
Präpositionalverwendung aufgrund ihrer starken Präsenz im täglich in Prag hörund lesbaren Deutsch schlichtweg nicht auffiel. Diese Vermutung wird durch die punktuelle Belegbarkeit ähnlicher an/auf-Schwankungen in Brods und Kischs Schriften gestützt:

$$
\begin{aligned}
& \text { an } \times \text { auf } \\
& \text { Über ihnen direkt schwebte die Decke und × Spiegel auf der Decke und zur Seite (Brod/ } \\
& \text { an ihr eine Posaune (Brod 1913: 120). Kafka 1987: 96). } \\
& \text { von klein an (Kisch 51922: 93). } \times \text { von Klein auf (Kisch }{ }^{51922: ~ 140) . ~}
\end{aligned}
$$

Hinzu kommen weitere Stellen bei Kisch, an welchen an und auf ebenfalls scheinbar verwechselt werden:

Sie hat solche rötliche Flecken an [,auf] der Haut (Kisch ${ }^{5}$ 1922: 129), einen teueren alten Ring auf [,an'] dem Finger (Kisch ${ }^{5}$ 1922: 14-15), Brillianten? Ja, so etwas funkelte auf [, an'] der linken Hand (Kisch ${ }^{5}$ 1922: 196).

Dass Prager Tageszeitungen (Bohemia) die von Kafka variierend eingesetzten Präpositionen ohne weiteres im Druck wiedergaben (D.412/5-7), verwundert nicht; denn auch im Deutsch der örtlichen Medien können - z. T. direkt nach tschechischem Muster - Besonderheiten festgestellt werden, die Kafkas anlaufSchwankungen ähneln:

auf [,für'] eine Stunde unterbrochen (PP 1921a: 4), die Halbzucker- [...] und Futterrübe auf [,zu'] Alkohol verarbeitet (PP 1921c: 8), auf [,an'] bestimmte Stunden gebunden (PP 1921c:15).

Weitere markante Parallelen zu tschechischen Sprachmustern in Kollokationen liegen in Phraseologismen vor, so z. B. in der Wendung, etwas zwischen vier Augen besprechen' (vgl. tsch. mezi čtyřma očima). In diesem Fall benutzte Kafka auch die gemeindeutsche Variante ,unter vier Augen'; eine Korrektur indiziert, dass er um die Korrektheit der letzteren wusste, die auch in den Referenzquellen konkurrenzlos ist (vgl. Kott 1880: 350; Herzer und Prach 1909b: 1205; Sterzinger 1916: 507; Siebenschein 1936-1938: 228); insofern dürfte es sich bei der Konstruktion mit zwischen tendenziell um eine Form der Prager städtischen Umgangs-, nicht aber der Standardsprache gehandelt haben. 
zwischen vier Augen

[bei meinem Gespräch [...], das jedenfalls nur zwischen 4 Augen geführt werden müsste] (Sv.137/13,26*-28*), denn das was sie zwischen vier Augen gesagt haben, ist eben auch nur zwischen vier Augen gesagt (P.156/14-15), Gleich nach der Begrüßung [...] bat er K. um ein kurzes Gespräch [zwischen] \{unter\} 4 $(U>A)$ ugen (Pv.119/4-6). $\times$ unter vier Augen

w(är>enn) ihre Angelegenheiten [...] nicht jetzt undzwar (un>au)sführlich [bespro] unter vier Augen besp\{r\}ochen werden müßten (P.186/8-10), bloß unter vier Augen (N1v.200/1-2), die Absicht, ihnen das alles unter 4 Augen zu sagen (Dv.129/6-7), nicht in einer Sitzung, sondern unter vier Augen (D.236/15).

Ähnlich verhält es sich mit dem Phraseologismus, sich auf die Lippen beißen` (im Sinne von ,sich beherrschen, eine Äußerung, Emotion u. Ä. zurückhalten'), neben dem Kafka nach dem tschechischen Muster kousnout se do rtu auch ,(sich) in die Lippen beißen' kannte - eine Variante, die zumindest bei Sterzinger (1931: 1690) als in Böhmen standardkonform belegt ist:

\section{sich in die Lippen beißen \\ $K$. biß in die Lippen vor Ungeduld \\ (S.189/20-21), die Mutter, [...] tränenvoll in die Lippen beißend (D.257/25-27). \\ sich auf die Lippen beißen \\ sagte der Offizier erstaunt und biß sich auf die Lippen (D.209/22-23), Der Reisende biß sich auf die Lippen (D.240/22-23).}

Des Weiteren findet sich im Korpus ein Einzelbeleg für die Wendung ,eine Prüfung aus etwas ablegen/machen': nach einer kurzen Prüfung aus der Religion (T.441/27-442/2). Auch diese Präpositionalverwendung, die im tschechischen vykonatldělat zkoušku z něčho ihre Entsprechung hat, war gemäß den Kodizes (vgl. Kott 1887: 509; Siebenschein 1944: 267) im böhmischen Standarddeutsch gängig, neben der heute gemeindeutschen Variante mit der Präposition in, die sich in Kafkas Autograph nicht findet.

\subsection{Unterdifferenzierung als Quelle semantischer Verschiebungen}

Wie schon die Interferenzen zwischen tschechisch na und deutsch an/ auf nahe legen, konnten sich v. a. in Bereichen, in welchen die beiden Sprachen Tschechisch und Deutsch gegenüber der jeweils anderen unterdifferenzierend sind, semantische bzw. phraseologische Verschiebungen in Kafkas Deutsch etablieren. Dieser Eindruck kann an einigen Beispielen schlaglichtartig erhärtet werden: Wenn von Anstrengung, die sie sich gaben (P.311/8-9), die Rede ist, wollte Kafka prinzipiell ausdrücken, dass sich jemand ,Mühe' gab. Auch wenn dem im Tschechischen kein Funktionsverbgefüge entspricht, ${ }^{1}$ so verweist das ungewöhnliche Substantiv doch auf die Synonyme námaha und úsili, welchen im Deutschen jeweils sowohl ,Anstrengung' als auch ,Mühe' entsprechen (vgl. Kott 1884: 422; Sterzinger 1916: 340; Herzer und Prach 1909b: 973; 1920: 1395; Siebenschein 1936-1938: 157).

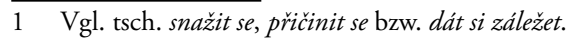


Kafkas Schwanken zwischen in und zu einem Zeitpunkt:

in einem solchen Zeitpunkt (V.29/14). $\quad$ × zu einem andern Zeitpunkt (P.268/24).

scheint demgegenüber auf den Umstand zurückführbar, dass Zeitpunkt und Augenblick im Tschechischen im Lexem okamžík zusammenfallen und hier die Bildung der Kollokation mit der Präposition $v$ (,in') erfolgt (vgl. Herzer und Prach 1909b: 1288). Als gruppenspezifisch erweist sich die Konstruktion u. a. dadurch, dass sie sich auch in Max Brods (1912: 175; 1916: 13) Prosa-Werken findet.

Wenn Kafka bei temporalen Abläufen mehrfach einer hinter dem andern schrieb, obwohl er kein räumliches, sondern das zeitliche Verhältnis ,einer nach dem anderen " ausdrücken wollte, so deutet sich auch hier eine Interferenz aus dem Tschechischen an, in dem das entsprechende Phrasem sowohl mit $z a$,hinter ${ }^{`}+$ Instr. (jeden $z a$ druhým) als auch mit po ,nach' + Lok. (jeden po druhém) gebildet werden kann (vgl. Sterzinger 1931: 342; Siebenschein 1944: 2):

um des Himmels willen nur immer einer hinter dem andern (S.378/4-5), Eine kleine Wandtür [...] wurde geöffnet und sich bückend kamen einer hinter dem andern die Prinzessin und der Prinz hervor (N2.238/23-25), Die vier andern verließen inzwischen mit zwei leichten Sprüngen einer hinter dem andern den Stall (T.513/3-5).

Das einzige Gegenbeispiel mit nach findet sich wohl nicht von ungefähr in der Leipziger Buchausgabe der Verwandlung, ging also womöglich auf das Korrektiv reichsdeutscher Verlagslektoren zurück: wie dann die Mutter auf den Vater zulief und ihr auf dem Weg die aufgebundenen Röcke einer nach dem anderen zu Boden glitten (D.171/20-23).

Mit dem Interesse, welches die ganze Straße an der Angelegenheit nahm, ging es Kafka zweifellos um den ,Anteil', der genommen wurde. Hier mag man an den tschechischen zájem denken, der als ,Interesse‘ wie ,Anteil' ins Deutsche übertragen werden kann (vgl. Kott 1887: 89; Sterzinger 1916: 343; 1921: 978; Herzer und Prach 1920: 1729), wie Kafkas Schwanken zwischen beiden Substantiven belegt:

\begin{tabular}{|c|c|}
\hline $\begin{array}{r}\text { Interesse nehmen } \\
\text { Interesse, welches die ganze Straße an der } \\
\text { Angelegenheit nahm (V.325/26-27). }\end{array}$ & $\begin{array}{l}\text { Anteil nehmen } \\
\text { [sogar Amalia nahm in ihrer Art Anteil] } \\
\text { (S.288/2-3,214*), Sie nehmen einen } \\
\text { Anteil an ihm, den er durchaus nicht } \\
\text { verdient (V.231/26-27), Ein guter } \\
\text { Deutscher [...] nimmt an allem [...] } \\
\text { einen merkwürdig freundlichen Anteil } \\
\text { (T.1038/15-19). }\end{array}$ \\
\hline
\end{tabular}


Die Wendung Interesse an etwas nehmen lässt sich dabei durch einen Eintrag bei Siebenschein (1939-1940: 463) sogar als regional normkonform belegen.

Wenn Kafka eine Handlungsweise als vorzeitig bezeichnete, obwohl er - wie an anderer Stelle - ,voreilig', d. h. ,zu schnell und unbedacht, unüberlegt', meinte, dann dürfte sich hier die Doppeldeutigkeit des tschechischen predčasný ausgewirkt haben, das sowohl für vorzeitig (,früher als vorgesehen, erwartet') als auch für voreilig im oben erläuterten Sinn stehen kann (vgl. Herzer und Prach 1916: 404; Sterzinger 1935: 1261, 1288):

[Wer sich nicht als Dichter erheben und $\times$ Ich war vielleicht voreilig (P.135/19). ihn blind und vorzeitig verurteilen würde]

(Pv.178/19).

Diese Doppelbedeutung von vorzeitig lässt sich u. a. in der Prager Presse als Form der Prager Schriftsprache nachweisen: Im Uebrigen ist diese Berechnung vorzeitig (PP 1921c: 7).

Ein weiterer Bohemismus des, Unterdifferenzierungstyps' findet sich nicht nur in den Prager Normkodizes, sondern auch in der Prosa Max Brods: Wenn Kafka einen Umstand als $z u$ kleinlich, um eine folgende Handlung vorzunehmen, bezeichnete, meinte er gemessen am Kontext, sie sei ,zu unbedeutend'. Nun kann das tschechische Adjektiv malicherný sowohl für ,kleinlich ' (bezogen auf eine Person) als auch für ,geringfügig' oder ,unbedeutend' (hinsichtlich eines Gegenstandes) stehen (vgl. Kott 1878: 969; Herzer und Prach 1909b: 800):

kleinlich ,geringfügig, unbedeutend" $E(r>s)$ schien ihm zu kleinlich, um ein Stückchen zu betteln (Vv.142/19), dazu ist die Sache doch zu kleinlich (P.43/3-4), [Wie kleinlich ist aller dieser Streit] (N2v.226/6), Kleinliche, ergebnislose schriftstellerische Arbeit (T.620/19). $\times$ kleinlich ,engstirnig, pedantisch' Diese\{s\} kleinliche Bedenken war daran schuld (N1.370/14-15), Jenseits des Platzes [...] schien mich jemand mit einem Operngucker zu beobachten(.>,) (a>w)ahrscheinlich weil ich ein neuer Mieter [hier] war(.>, ) [Nun fand ich] das war kleinlich (N2v.243/21-24), daß auch hier die rechnerische Arbeit nicht kleiner sondern nur kleinlicher war (N2.301/13-14)., Das war kleinliche Narrheit (T.381/8), alles was ich täte wäre kleinlich (T.528/17).

Die Mehrzahl der herangezogenen Prager Normkodizes weist kleinlich in beiden Bedeutungen aus (vgl. Kott 1878: 969; Herzer und Prach 1909b: 800; Sterzinger 1921: 429, 1208; Siebenschein: 1939-1940: 591); und in Brods literarischen Schriften lässt sich die doppelte Semantik sogar innerhalb eines Werkes nachweisen: 
kleinlich ,geringfügig, unbedeutend ${ }^{\prime} \times$

meine Sehnsucht, irgendein Ding [...] durch Ordnung zu beherrschen, über seine

kleinlichsten Veränderungen zu wachen kleinlich ,engstirnig, pedantisch'

mit Menschen [...] derart kleinlich und mißverständlich verfahren (Brod 1913: 165).

Schließlich sei eine weitere semantisch-phraseologische Verschiebung erwähnt, die zwar nicht in Kafkas Prosa, jedoch in den örtlichen Printmedien vorkommt: Vor dem Hintergrund von tsch. počitat, dem sowohl ,rechnen“ als auch ,zählen“ entsprechen, bildete man in der Prager Presse die Wendung, mit etwas rechnen' mit dem Verb zählen: indem sie mit einer unmittelbaren bewaffneten Hilfe Westeuropas zählten (PP 1921c: 3). Im Tschechischen entspräche dem die Konstruktion počitat s někým/něčim. Auch diese Schwankung darf diachron als regional schriftsprachlich akzeptiert gelten, zumal die Wendung mit etwas zählen in immerhin einem der Referenzwörterbücher genannt wird (vgl. Kumprecht ${ }^{3}$ 1940: 146, 275).

\subsection{Phrasenmuster nach tschechischem Vorbild}

Hinzu kommen Sonderformen im Bereich der Phraseologie, die Analogien zu tschechischen Mustern aufweisen, teils neben gemeindeutschen Varianten und teils in den Kontrollkorpora als standardkonform oder -nahe belegt. Dass Brod diese Formen unverändert abdruckte, ${ }^{1}$ verdeutlicht, dass sie auch seinem Prager Sprachgefühl nach ,richtig' und daher in seinem Umfeld allgemein gebräuchlich waren:

Da ich aber schon um fünf [...] wieder auf dem Platze sein muss (V.176/20-22), Wenn nur der Heizer besser auf dem Platze gewesen wäre (V.32/24-25), ${ }^{2}$ Mir ist es nicht traurig (P.131/26-27), war mir alles so traurig (V.300/3), ${ }^{3}$ soweit es in seinen Kräften $(z u>w)$ ar (Pv.116/20), ${ }^{4}$ wenn es einem noch so sehr gegen den Sinn geht (P.160/15), ${ }^{5}$ daraus eine Lehre [...] nehmen (P.258/8), ${ }^{6}$ der Diener [...] glaubte, er sei dort [...] nicht mehr am Platze (V.22/7-9), [ [man hatte sich [...] nicht einmal die Mühe genommen sie zuzumachen]

1 Vgl. Kafka (1953: 21, 106; 1964: 439; 1965: 85, 110, 123, 146, 178, 227).

2 Vgl. tsch. být na svém mistě (eigentlich ,auf seinem Posten sein'); Siebenschein (1944: 212) führt immer auf dem Platz sein als normkonforme Konstruktion an.

3 Die tsch. Wendung je mi smutno (eigentlich, ich bin traurig') gibt Hulík (1936: 539) als umgangssprachliche Form für dt. mir ist bange an; in Kafkas Autograph lässt sich auch die gemeindeutsche Variante belegen: Herr Polunder war traurig geworden (V.72/17-18).

4 Vgl. tsch. co bylo v jeho moci (eigentlich, was in seinen Kräften stand); die Prager Wörterbücher kennen die Wendung in seinen Kräften sein nicht (vgl. Kott 1878: 1052; Herzer und Prach 1909b: 883; Sterzinger 1921: 1369; Siebenschein 1939-1940: 662).

5 Vgl. tsch. když je mu to proti mysli (eigentlich, wenn es ihm gegen den Strich geht'); gegen den Sinn gehen ist in den Prager Kodizes nicht belegt (vgl. Kumprecht ${ }^{3}$ 1940: 92; Siebenschein 1944: 825).

6 Vgl. tsch. vzit si z toho poučení (eigentlich, daraus eine Lehre ziehen'); eine Lehre aus etwas nehmen findet sich in den Referenz-Wörterbüchern (vgl. Kott 1880: 87; Sterzinger 1931: 1594; Siebenschein 1939-1940: 744).

7 Vgl. tsch. neni tam na misté (eigentlich,er ist dort fehl am Platze); die Wendung nicht auf dem Platz sein fehlt in den Nachschlagewerken (vgl. Herzer und Prach 1916: 863; Sterzinger 1931: 800; Siebenschein 1944: 212). 
(Sv.370/12), ohne [mir] \{sich\} aber die Mühe zu nehmen, einen verständlichen Grund für $(m>s)$ eine Bitte zu erfinden (Sv.55/2-3(2)), [wenn dieser [\{sich\}] nicht etwa [die Mühe nahm] \{Freundlichkeit hatte $\}$ sich nach dem Besucher umzuwenden] (Pv.140/16). ${ }^{1}$

Im Prager Tagblatt schließlich erscheint die an sich für das Tschechische exklusive Redewendung prijicit s križkem po funuse, d. h. ,zu spät, nach Torschluss kommen“ (vgl. Kott 1878: 378; Herzer und Prach 1909a: 302), wörtlich ins Deutsche übertragen: Der „Čas" [eine Zeitung] kommt mit dem Kreuz nach dem Begräbnis (PT 1921: 4). Auch hier spricht das Vorkommen im Kontrollkorpus für die prinzipielle Schriftsprachlichkeit der Wendung in Prag.

\subsection{Substituierung von Verben durch das, tschechische' geben}

Darüber hinaus illustrieren einige für Kafkas Deutsch typische Wendungen durchaus die von Egon Erwin Kisch (1992: 251-252) im ,Kleinseitner Deutsch ausgemachte, für das Tschechische charakteristische Substituierung verschiedener Verben durch dát (,geben'): Im Verschollenen etwa ,geben sich' die Romanfiguren nicht nur Anstrengung, sondern auch das Rendezvous, anstatt es zu ,verabreden'; aufwändige Tätigkeiten ,machen', ,verursachen' oder ,bereiten' ihnen keine Arbeit oder Mühe, sondern, geben'sie ihnen; auch die Verben in den Funktionsverbgefügen ,jemandem zu schaffen machen', ,eine Aufgabe stellen' und ,sich die Mühe machen werden im Korpus durch geben ersetzt:

das Rendezvous, das wir uns beim Haupttor gegeben hatten (V.262/27-263/1), Sie gibt mir ein Rendezvous für den nächsten Tag (T.1029/12-13), ${ }^{2}$ welche Arbeit es gegeben hat, diese Sachen die Treppe h(in>er)aufzutragen (Vv.311/16-17), die gewöhnlichsten Fälle [...] geben noch viel mehr und freilich auch viel ergiebigere Arbeit (S.107/14-16), Das Hineinheben Bruneldas gab noch eine gewisse Arbeit (V.378/2-3), Die Verwaltung aller [Wasser-] | Gewässer gab ihm unendliche Arbeit (N2v.300/22-23), Das gibt gar keine Mühe (N2.274/13), so daß es mir immer nur wenig Mühe gegeben hätte (N2.577/8-9), ${ }^{3}$ Die Liftjungen geben mir aber heute zu schaffen (V.235/13-14), ${ }^{4}$ die Aufgabe, die er selbst

1 Vgl. tsch. brátlvzit na sebe prácilnámahu (eigentlich ,sich die Arbeit/Mühe machen'); die Wörterbücher führen sich die Mühe geben nicht an (vgl. Sterzinger 1935: 1067; Siebenschein 1939-1940: 915); unter Kafkas Varianten findet sich u. a. Machen Sie sich keine Mühe (D.35/11-12).

2 Vgl. tsch. dát si rande; die Wendung jemandem/sich ein Rendezvous geben lässt sich bei Kott (1878: 286), Sterzinger (1931: 1092) und Siebenschein (1944: 378) als standardkonform nachweisen; auch in Max Brods Reisetagebüchern ist sie belegt: wo offen die Deutschen bei deutschen Kellnern und Zeitungen sich ein Rendezvous geben (Brod/Kafka 1987: 119).

3 Vgl. tsch. něco dá něčemu mnoho práce bzw. něco dá namáhání; zumindest Sterzinger (1931: 291) rechnet die Variante etwas gibt Mühe der Schriftnorm zu.

4 Vgl. tsch. dát komu mnoho práce; die Variante jemandem zu schaffen geben findet sich bei Sterzinger (1935: 87) und Siebenschein (1944: 479) als Teil des Standards verzeichnet. 
gegeben hatte (N2.550/24-25), ${ }^{1}$ Klamm würde sich gar nicht die Mühe geben erst einen Brief zu schreiben $(\mathrm{S} .310 / 1-2) .^{2}$

Die Prager Referenzquellen gehen auch bei diesen Konstruktionen in den meisten Fällen von ihrer Normkonformität aus; z. T. finden sie sich auch in Max Brods Schriften belegt (vgl. Brod/Kafka 1987: 119).

\section{Zusammenfassung}

Resümiert man die bis hierher gemachten Befunde, so kommt man zu folgendem Ergebnis: Kafkas Schriftdeutsch weist erheblich mehr Bohemismen auf als von der Wissenschaft bisher angenommen. Ein Einwirken des Tschechischen bzw. der sprachenkontaktlastigen deutschen Soziolekte (,Prager', ,Kleinseitner Deutsch') der Prager Unterschichten auch auf die Sprache des Prager deutschsprachigen Bürgertums ist damit erwiesen; zugleich lässt sich das durch Max Brod, Johannes Urzidil u. a. ex post verbreitete Autostereotyp, die Prager (jüdisch-)deutschen Schriftsteller seien exklusiv im Besitz eines überregionalen, ,reinen' Standarddeutsch, als kollektive Wunsch-Projektion entlarven. Tschechische Sprachstrukturen manifestieren sich in erster Linie in der Syntax, der Phraseologie und der Idiomatik von Kafkas Deutsch, wobei v. a. die Unterdifferenzierung des Tschechischen gegenüber dem Deutschen in manchen Bereichen semantische Verschiebungen bewirkt hat. Dass es sich in der Masse nicht etwa um Facetten des kreativen Umgangs des Autors mit der Sprache handelt, sondern um Elemente seiner authentischen Sprachverwendung, ließ sich dadurch nachweisen, dass gleiche oder ähnliche Phänomene auch in Texten von Schriftstellern seines engeren Sozialisationsmilieus oder in zeitgenössischen Prager Presseerzeugnissen vorkommen. Diese tschechischen Muster sind somit - als Resultate des deutsch-tschechischen Sprachenkontakts - als in Prag oder sogar ganz Böhmen gängige, kollektiv verwendete Varianten und damit als gruppenspezifisch zu werten. Die Belegbarkeit auch in Prager Standard-Wörterbüchern weist viele dieser Bohemismen darüber hinaus als Teil eines besonderen Prager (oder böhmischen) Standards des Deutschen aus, der sich punktuell nicht nur vom zeitgenössischen ,reichsdeutschen', sondern auch vom schulisch vermittelten gesamtösterreichischen Standard der Zeit unterschied.

1 Vgl. tsch. dát komu co za úlohu, dát komu úkol; Kafkas Variante wird von den Prager Referenzkodizes nicht angeführt (vgl. Kott 1884: 336, 351; Sterzinger 1916: 438; Herzer und Prach 1920: 1364; Siebenschein 1936-1938: 199).

2 Vgl. tsch. dát si záležet; in den Referenz-Wörterbüchern fehlt sie Wendung sich die Mühe geben (vgl. Sterzinger 1916: 782-783; Siebenschein 1939-1940: 915). 


\section{Literaturverzeichnis}

Ammon, Ulrich (2005): Standard und Variation: Norm, Autorität, Legitimation. In: Eichinger, Ludwig M. / Kallmeyer, Werner (Hg.): IDS-Jahrbuch Standardvariation. Wie viel Variation verträgt die deutsche Sprache? Berlin / New York, de Gruyter. S. 28-40.

Bednarský, Petr (2002): Deutsche und tschechische Präpositionen kontrastiv - am Beispiel von an, auf und na (= Mehrsprachigkeit 11). Münster, Waxmann.

Beißner, Friedrich (1952): Der Erzähler Franz Kafka. Ein Vortrag. Stuttgart, Kohlhammer.

Binder, Hartmut (1996): Entlarvung einer Chimäre: Die deutsche Sprachinsel Prag. In: Godé, Maurice / Le Rider, Jacques / Mayer, Françoise (Hg.): Allemands, Juifs et Tchèques à Prague de 1890 à 1924 / Deutsche, Juden und Tschechen in Prag 18901924 (= Bibliothèque d'Études Germaniques et Centre-Européennes 1). Montpellier, Université Paul-Valéry. S. 183-209.

Blahak, Boris (2014a): „Ein Tscheche? Nein.“ Franz Kafkas topographisch bedingte Exophonie und die innerste Dyade von Judentum und Slavia. In: Smola, Klavdia / Terpitz, Olaf (Hg.): Jüdische Räume und Topographien in Ost(mittel)europa. Konstruktionen in Literatur und Kultur (= Opera Slavica. Neue Folge 61). Wiesbaden, Harrassowitz. S. 23-55.

Blahak, Boris (2014b): Das „Reichsdeutsche“ als prestigeträchtige Zielnorm in Prager deutschen Schriftstellerkreisen im frühen 20. Jahrhundert. Das Beispiel Franz Kafka. In: Šemelík, Martin (Hg.): Germanistica Pragensia XXIII (= Acta Universitatis Carolinae. Philologica 2). Festschrift für Eva Berglová. Praha, Univerzita Karlova / Karolinum. S. 23-58.

Blahak, Boris (2015): Franz Kafkas Literatursprache. Deutsch im Kontext des Prager Multilingualismus (= Intellektuelles Prag im 19. und 20. Jahrhundert 7). Wien / Köln / Weimar, Böhlau.

Braese, Stephan (2010): Eine europäische Sprache. Deutsche Sprachkultur von Juden 1760-1930. Göttingen, Wallstein.

Brod, Max (1911): Jüdinnen. Berlin, Juncker.

Brod, Max (1912): Arnold Beer. Das Schicksal eines Juden. Berlin / Charlottenburg, Juncker.

Brod, Max (1913): Über die Schönheit häßlicher Bilder. Ein Vademecum für Romantiker unserer Zeit. Leipzig, Wolff.

Brod, Max (1916): Die erste Stunde nach dem Tode. Eine Gespenstergeschichte. Leipzig, Wolff.

Brod, Max ( $\left.{ }^{3} 1954\right)$ : Franz Kafka. Eine Biographie. Frankfurt a. M., Fischer.

Brod, Max (1960): Streitbares Leben. Autobiographie. München, Kindler.

Brod, Max (1969): Streitbares Leben. 1884-1968. München / Berlin / Wien, F. A. Herbig.

Brod, Max (1974): Über Franz Kafka. Frankfurt a. M., Fischer Taschenbuch.

Brod, Max / Kafka, Franz (1987): Eine Freundschaft I. Reiseaufzeichnungen. Hg. von Malcolm Pasley und Hannelore Rodlauer. Frankfurt a. M., Fischer.

Cohen, Gary B. (1981): The politics of ethnic survival: Germans in Prague 1861-1914. Princetown, University Press.

Dietz, Ludwig (1963): Franz Kafka - Drucke zu Lebzeiten. In: Jahrbuch der deutschen Schillergesellschaft 7/1963. S. 416-457.

Eisner, Pavel (1946): Chrám i tvrz. Kniha o češtině. Praha, Jaroslav Podroužek.

Herzer, Jan / Prach, Adalbert (1909-1920): Českonémecky slovnik. Böhmischdeutsches Wörterbuch. 4 Bde. [Bd. 1: 1909a; Bd. 2: 1909b; Bd. 3: 1916; Bd. 4: 1920]. Praha, J. Otto.

Hesse, Hermann (1987): Schriften zur Literatur 2 (= Hermann Hesse. Gesammelte Werke. Bd. 12). Frankfurt a. M., Suhrkamp. 
Hulík, Vojtěch (1936): Česko-německý slovnik živé mluvy s frázemi a gramatikou pro školy i soukromnou potrebu. Tschechisch-deutsches Wörterbuch der Umgangssprache mit Phrasen und Grammatik für Schule und Haus. Praha, Školní nakladatelství.

Kafka, Franz (1953): Amerika. Roman. Hg. von Max Brod. Frankfurt a. M., Fischer.

Kafka, Franz (1964): Das Schloß. Roman. Hg. von Max Brod. Frankfurt a. M., Fischer.

Kafka, Franz (1965): Der Prozess. Roman. Hg. von Max Brod. Frankfurt a. M., Fischer.

Kafka, Franz (1974): Briefe an Ottla und die Familie. Hg. von Hartmut Binder und Klaus Wagenbach. Frankfurt a. M., Fischer.

Kafka, Franz ( $\left.{ }^{2} 1983\right)$ : Das Schloß. Hg. von Malcolm Pasley. 2 Bde. Frankfurt a. M., Fischer. Kafka, Franz (1990a): Der Proceß. Hg. von Malcolm Pasley. 2 Bde. Frankfurt a. M., Fischer.

Kafka, Franz (1990b): Tagebücher. Hg. von Hans-Gerd Koch, Michael Müller und Malcolm Pasley. 3 Bde. Frankfurt a. M., Fischer.

Kafka, Franz (1992): Nachgelassene Schriften und Fragmente II. Hg. von Jost Schillemeit. 2 Bde. Frankfurt a. M., Fischer.

Kafka, Franz (1993a): Nachgelassene Schriften und Fragmente I. Hg. von Malcolm Pasley. 2 Bde. Frankfurt a. M., Fischer.

Kafka, Franz (1994/1996): Drucke zu Lebzeiten. Hg. von Wolf Kittler, Hans-Gerd Koch und Gerhard Neumann. 2 Bde. Frankfurt a. M., Fischer.

Kafka, Franz (2002): Der Verschollene. Hg. von Jost Schillemeit. 2 Bde. Frankfurt a. M., Fischer Taschenbuch.

Kisch, Egon Erwin (1917): Prager Deutsch. In: Deutsche Zeitung Bohemia 90/282/1917 (14.10., Morgen-Ausgabe). S. 3-4.

Kisch, Egon Erwin (1922): Soldat im Prager Korps. Leipzig / Prag, Verlag der K. Andréschen Buchhandlung.

Kisch, Egon Erwin ( $\left.{ }^{5} 1922\right)$ : Der Mädchenhirt. Berlin, Reiß.

Kisch, Egon Erwin (1992): Die Abenteuer in Prag. Bd. 1. Berlin, Aufbau.

Kott, František Št. (1878-1893): Česko-německý slovník zvlaště grammaticko-fraseologický. 7 Bde. [Bd. 1: 1878; Bd. 2: 1880; Bd. 3: 1882; Bd. 4: 1884; Bd. 5: 1887; Bd. 6: 1890; Bd. 7: 1893]. Praha, Josef Kolař.

Kranzmayer, Eberhard (1956): Historische Lautgeographie des gesamtbairischen Dialektraumes. Wien, Österreichische Akademie der Wissenschaften.

Kučera, Petr (2014): Franz Kafka a problém identity. In: Pospíšil, Ivo / Zelenka, Miloš: Kontexty literárni védy IV. Brno, Tribun EU. S. 65-86.

Kučera, Petr (2013): The Disappearance of Identity in Franz Kafka's Prose Metamorphosis. In: Mišterová, Ivona / Skopečková, Eva et al.: A Search for Identity. Plzeň: Západočeská univerzita. S. 99-122.

Kumprecht, Karel ( $\left.{ }^{3} 1940\right):$ Malý némecko-český slovník unikum. Praha, A. Neubert.

Kummer, Karl Ferdinand ( $\left.{ }^{3} 1892\right)$ : Deutsche Schulgrammatik. Prag / Wien, F. Tempsky Leipzig, G. Freytag.

Martini, Fritz (1958): Ein Manuskript Franz Kafkas: Der Dorfschullehrer. In: Jahrbuch der Deutschen Schillergesellschaft 2/1958. S. 266-300.

Nekula, Marek (2004): Kafkas Verwendung der deutschen Sprache. In: Kafka Katern 12/4/2004. S. 93-97.

Pasley, Malcolm ( $\left.{ }^{2} 1983\right)$ : Entstehung. In: Kafka, Franz: Das Schloß. Hg. von Malcolm Pasley. Apparatband. Frankfurt a. M., Fischer. S. 59-89.

Pick, Otto (1913): Die Probe. Novellen. Heidelberg, Meister.

PP (1921a): Prager Presse 1/5/1921 (01.04., Morgen-Ausgabe).

PP (1921b): Prager Presse 1/6/1921 (02.04., Abend-Ausgabe).

PP (1921c): Prager Presse 1/7/1921 (03.04., Morgen-Ausgabe). 
PT (1921): Prager Tagblatt 46/1/1921 (01.01.).

Rinas, Karsten (2003): Vorsicht - Fehler! Odstraňujeme nejčastějši „české“ chyby v němčině. Plzeň, Fraus.

Ritschel, Augustin (1893): Das Prager Deutsch. In: Phonetische Studien 2/1893. S. 129-133.

Schillemeit, Jost (2002): Arbeitsweise. In: Kafka, Franz: Der Verschollene. Hg. von Jost Schillemeit. Apparatband. Frankfurt a. M., Fischer Taschenbuch. S. 82-89.

Schleicher, August (1851): Ueber die wechselseitige Einwirkung von Böhmisch und Deutsch. In: Archiv für das Studium der neueren Sprachen und Literaturen 6/9/1851. S. 38-42.

Siebenschein, Hugo (1936-1948): Přručni slovník německo-český. Deutsch-tschechisches Handwörterbuch. 4 Bde. [Bd. 1: 1936-1938; Bd. 2: 1939-1940; Bd. 3: 1944; Bd. 4: 1944-1948]. Praha, Státní nakladatelství.

Skála, Emil (1977): Der deutsch-tschechische Bilinguismus. In: Moser, Hugo / Eggers, Hans / Erben, Johannes / Leys, Odo / Neumann, Hans (Hg.): Sprachwandel und sprachwissenschaftliche Geschichtsschreibung im Deutschen. Düsseldorf, Schwann-Bagel. S. 260-279.

Skála, Emil (1991): Das Prager Deutsch. In: Jahrbuch der Bayerischen Akademie der schönen Künste 5/1991. S. 130-140.

Stein, Herbert von (1976): Betrachtungen über das ,Prager Deutsch'. In: Prager Nachrichten 27/5-6. S. 13-15.

Steinig, Wolfgang (1980): Zur sozialen Bewertung sprachlicher Variation. In: Cherubim, Dieter (Hg.): Fehlerlinguistik. Beiträge zum Problem der sprachlichen Abweichung (= Germanistische Linguistik 24). Tübingen, Niemeyer. S. 106-123.

Sterzinger, Josef V. (1916-1935): Encyklopedický nèmecko-český slovnik. Deutsch-böhmisches Wörterbuch. 4 Bde. [Bd. 1: 1916; Bd. 2: 1921; Bd. 3: 1931; Bd. 4: 1935]. Praha, J. Otto.

Štícha, František (2003): Česko-německá srovnávaci gramatika. Praha, Argo.

Thieberger, Richard (1979): Sprache. In: Binder, Hartmut (Hg.): Kafka-Handbuch in zwei Bänden. Bd. 2. Das Werk und seine Wirkung. Stuttgart, Alfred Kröner. S. 177-203.

Tucholsky, Kurt (1962): Ausgewählte Briefe 1913-1935. Hg. von Mary Gerold-Tucholsky und Fritz J. Raddatz. Reinbek b. Hamburg, Rowohlt.

Urzidil, Johannes (1972): Bekenntnisse eines Pedanten. Erzählungen und Essays aus dem autobiographischen Nachlaß. Zürich / München, Artemis.

Uyttersprot, Herman (1957): Eine neue Ordnung der Werke Kafkas? Zur Struktur von ,Der Prozeß' und,Amerika: Antwerpen, De Vries-Brouwers.

Ziegler, Evelyn (1999): Deutsch im 19. Jahrhundert: Normierungsprinzipien und Spracheinstellungen. In: Bister-Broosen, Helga (Hg.): Beiträge zur historischen Stadtsprachenforschung (= Schriften zur diachronen Sprachwissenschaft 8). Wien, Praesens. S. 79-100.

\author{
Boris Blahak \\ Ústav germánských studií \\ Filozofická fakulta Univerzity Karlovy \\ Nám. Jana Palacha 2 \\ 11638 Praha 1
}

